\title{
The "S" in Social Network Games: Initiating, Maintaining, and Enhancing Relationships
}

\author{
Donghee Yvette Wohn \\ Michigan State University \\ wohndong@msu.edu
}

\author{
Cliff Lampe \\ Michigan State University \\ lampecli@msu.edu
}

\author{
Rick Wash \\ Michigan State University \\ wash@msu.edu
}

\author{
Nicole Ellison \\ Michigan State University \\ nellison@msu.edu
}

\author{
Jessica Vitak \\ Michigan State University \\ vitakjes@msu.edu
}

\begin{abstract}
Social network games embedded within social network sites (SNSs) such as Facebook facilitate play with "Friends" within the SNS. In this study, we look at different dimensions of how game play contributes to relationship initiation and development using qualitative data collected from adult Facebook users $(N=18)$. Our data suggest that interpersonal motivations are a primary driver of initial game play and that while game play doesn't facilitate direct social interaction, participants perceive indirect interaction and sharing game-based content as useful in maintaining and even enhancing relationships.
\end{abstract}

\section{Introduction}

The popularity of social network sites (SNSs) has provided a vibrant platform for scholars who are looking beyond the question of whether or not SNSs are social to the ways in which they support sociality $[12,18,28,31]$. As the largest SNS in the United States [13] with more than 400 million users worldwide, Facebook has been one of the most widely studied SNSs to date. While Facebook started in post-secondary institutions in the U.S., it has since become available to, and adopted by, more diverse users, encompassing wider demographics including age. By $2009,39 \%$ of Internet-using U.S. adults age 30 or older reported using at least one SNS, and $75 \%$ of this group had a Facebook profile [20].

In order to understand how adult users are using Facebook for social purposes, we conducted in-depth interviews with 18 Facebook users ages 25 to 55 . During the course of these interviews and in subsequent analyses of the data, we identified a clear theme whereby participants described the various ways that game applications available on Facebook affected their relationships with other Facebook users.

Our findings suggest that participants perceived three outcomes of their social game use on their social relationships: maintaining, initiating, and enhancing relationships. These were recurring themes among our participants. We describe how participants talked about using the games to facilitate different types of relationships and the features that were important for these processes.

\section{Relationships in SNSs}

There has been considerable work on how use of social network sites (SNSs) affect relationships, including the impact of Facebook on college students' social capital [12,18,28], demographic characteristics of users [14], concepts of friendship in SNSs [21], and motivations of SNS use $[16,25,26]$.

Researchers have also examined various aspects of interpersonal relationships on Facebook. Scholars have identified how Facebook is used to initiate new relationships and maintain old ones $[6,12]$; they found that it is more often used to articulate previously established relationships than for connecting with strangers.

Researchers have also looked at how SNS use affects social relationships in non-student populations. Burke and colleagues [5] looked at Facebook users of wide demographic variety to see how time spent on the site and type of use affected different types of social capital and psychological outcomes. Using survey responses and server level data, they found that passive use of the site (viewing content, but not contributing) increases 
loneliness, while active use of the site (viewing and contributing content) decreases loneliness.

SNSs also have the capacity to support relationship development within organizations. In work on IBM employees' use of Beehive, Steinfield and colleagues [32] found that more use of the SNS was associated with a stronger sense of connection to other organizational members. Skeels and Grudin [30] studied SNS users in a large company and found that a challenge for users was balancing relationships between work connections and personal connections, referred to sometimes as "context collapse."

Overall, SNSs have been shown to have an effect on how adults, in organizations and out, form and express their relationships. However, different features of SNSs may have different capacities for leading to these outcomes. SNSs can be thought of as bundles of features, and different features may affect relationship development and maintenance in varying ways, just as the affordances of different information communication technologies affect different uses [27]. For example, Joinson [16] found that there were different motivations to use Facebook, often tied to features like photo albums or private messaging. Chen et al. [6] found that tools like recommenders could change how people made connections in organizationally based SNSs.

Based on our interviews, it was clear that social games were being used in Facebook to manage relationships, but which relationships, and how?

RQ1: How are Facebook users using SNGs for relationship management?

RQ2: What types of relationships are being facilitated by SNGs?

\section{Method}

We conducted semi-structured interviews with 18 U.S. adults who had an active account on Facebook between November 2009 and January 2010. Participants were recruited through a series of Facebook ads that were shown to users between the ages of 25 and 55 in order to attract participants from a wide age range. The ad contained a link to a brief online screening survey about their general Facebook use, which asked about their age, ethnicity, length of Facebook membership, gender, and items pertaining to motivations for using Facebook. Participants who were interested in a 45-minute phone interview provided their email address; we contacted them individually to schedule an interview time.

Thirteen participants were recruited through the Facebook ads and an additional five were recruited through the use of snowball sampling, whereby participants were asked to forward an email about the study to any friends who they thought would be interested in participating and met the participation requirements (i.e., Facebook users age 25-55).

Table 1 describes the demographics of the participants, including the number of Facebook Friends for each participant. Our participants were all adult Facebook users. They were predominantly white and predominantly female, though we did get some meaningful variation along these dimensions. The size of the participants' networks (\# of Friends) varied widely, from as few as 16 Friends to over 700 Friends. This suggests that we interviewed users who represent a variety of different types of uses of Facebook.

Table 1. Participant demographics

\begin{tabular}{|l|l|l|l|l|}
\hline & Age & Sex & Ethnicity & $\begin{array}{l}\text { No. of } \\
\text { Facebook } \\
\text { Friends }\end{array}$ \\
\hline P1 & 45 & Male & White & 30 \\
\hline P2 & 41 & Female & White & 339 \\
\hline P3 & 48 & Male & Latino & 200 \\
\hline P4 & 51 & Female & Black & 164 \\
\hline P5 & 55 & Female & White & 300 \\
\hline P6 & 39 & Female & White & 125 \\
\hline P7 & 41 & Male & White & 750 \\
\hline P8 & 51 & Female & White & 139 \\
\hline P9 & 36 & Male & White & 40 \\
\hline P10 & 34 & Male & White & 25 \\
\hline P11 & 52 & Female & White & 30 \\
\hline P12 & 55 & Female & White & 50 \\
\hline P13 & 36 & Female & White & 30 \\
\hline P14 & 28 & Female & Black & 16 \\
\hline P15 & 27 & Male & Latino & 700 \\
\hline P16 & 51 & Female & White & 99 \\
\hline P17 & 39 & Female & Other & 250 \\
\hline P18 & 55 & Male & White & 120 \\
\hline
\end{tabular}

In the initial few interviews, we focused on general questions about Facebook use as well as specific questions about relationship management. The protocol initially did not have any gamespecific questions about relationship management. However, after initial interviews where participants introduced the concept of game play in response to more general questions about Facebook use (such as which features they used to communicate with certain people), we added a few questions to probe the phenomena more thoroughly. Examples of game-specific questions in the protocol include, "Do you play games on Facebook?" and "Do you play games with Facebook users who are not your friends?" 
The interviews were audio-recorded, transcribed by a research assistant, and checked for accuracy. We then conducted textual data analysis using Atlas.ti, a qualitative content analysis software program. In analyzing the interviews, we took an exploratory approach to answer generic questions about how Facebook users played Social Network Games (SNGs) and their relationships with other game players. The analysis was an iterative process, whereby we went through the transcripts to identify references to how Facebook affected their relationships with other users [9]. Separately, we coded all references about Facebook games.

Relationship management emerged as an important theme during coding. We examined all references about Facebook games and extracted comments that pertained to relationship management. These comments were arranged in a data matrix to better understanding of recurring themes [23]. The code identifying each participant was put in the vertical axis, and themes such as "initiating," "maintaining," and "enhancing" were placed in the horizontal axis. Themes for the horizontal axis were based on codes we had made about relationship management before looking specifically at the instances within games. The data matrix supported our efforts to check results for representativeness and conduct negative case analysis [24].

The themes were then developed through an iterative process of categorizing by combining certain codes together and creating new ones when it aided understanding of the data. Finally, in developing our narrative, we checked for negative cases as we wrote up the description of each major theme, and checked each quote for representativeness within the theme.

\section{Social network games}

Social Network Games (SNGs) are game applications made available through SNSs, where users play the game with members of their social network, as articulated on the site. In 2005, Facebook made an open API available for third party developers to create applications that could be accessed on Facebook as a platform. Many of the applications that were developed were games, which often took advantage of the social features of SNSs, including access to a list of Facebook Friends.

SNGs are a type of networked game. Although computer games were traditionally considered a solitary medium that instigates unsocial behavior [8], networked games help form a new form of social phenomenon. [33]
Socializing has been identified as being a reason why people play computer games. In one of the earliest categorization of game players, Bartle [4] identified "socializers" as one of the four player types found in Multi-User Dungeons (MUDs). Subsequent studies categorizing player types for video games in general [29] and Massively Multiplayer Online games (MMOs) [36] also show that socializing is a prominent characteristic of game players.

Much of the research on socialization in games looks at MMOs. Williams and colleagues [34] found that guild play in World of Warcraft was like a team sport, but even within the rules and social norms of the game, there were self-initiated tactics, styles, and goals that fostered socializing among players that often had little to do with the original game [7].

Social network games (SNGs) differ from traditional networked games because one must be "Friends" with other players within the SNS before being able to play games with them. [35] (We capitalize the word Friend to distinguish between friends in the colloquial sense and friends in Facebook.) This is slightly different from other multiplayer games such as MMOs, in which the technical "Friending" of other players is not a prerequisite of game play. SNG players can also create contact lists for their in-game network, which we will label in this paper as "Neighbors." Consequently, SNSs can host games that are SNGs and those that are single player only. In this work, we focus on the former.

What constitutes a SNG is determined more by technical aspects of how it is accessed and distributed, not on the genre of the game. In fact, there are many different genres in SNGs, including board, word, simulation, arcade, role-playing, and action games (see Fig. 1).

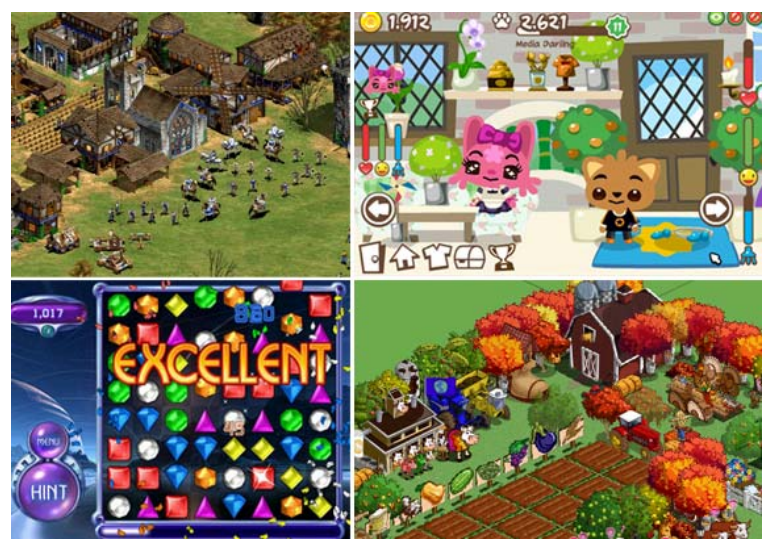

Figure 1. Different types of SNGs 
Some SNGs are closer to traditional board games or early computer games that were based on taking turns. For instance, Scrabulous (a version of Scrabble that was one of the early popular SNGs) [22] is entirely turn-based, where one player cannot play until another has made a move. Other SNGs, however, support multiplayer play that is asynchronous, but not turn-based; each player can play at his or her own pace and still be able to interact with other players. Many games, especially those that are the most popular applications on Facebook [3], are largely built on this mechanism of mixed cooperative and solitary play.

Due to the varying genres and styles, it is difficult to define SNGs by the content of the game. Rather, it is the connection with the SNS-being an application within a SNS and the use of the features of an existing SNS - that makes SNGs different from other games. Kirman and colleagues [17] tested the effects of social game play on the structure of social interactions on Facebook and found that providing information about the structure of the network increased engagement in the game play.

Wohn et al. [35] identified publishing one's game achievements as one of the seven types of SNG uses. Publishing is a feature that is unique to SNGs because the game applications have access to the players' Friends database and create a semiautomatic feature where the game prompts users to post their game achievements to their Wall (Fig.2). This Wall posting then appears to all of the players' Friends unless players have separate privacy settings.

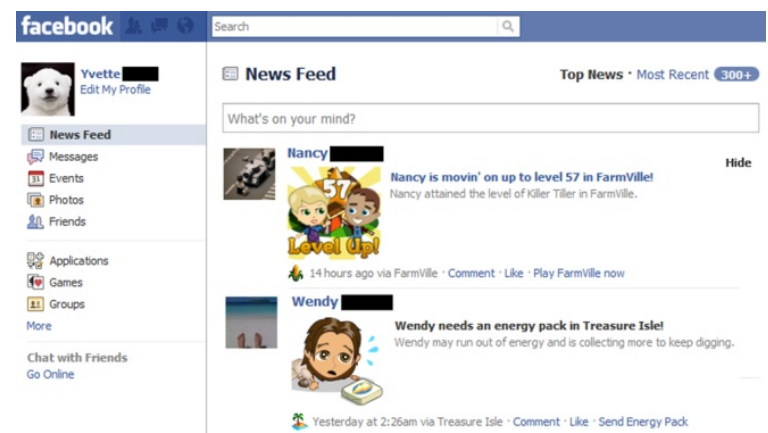

Figure 2. Friends' game status updates appearing in the News Feed

\section{Social play}

Fifteen of the 18 participants played games on Facebook, but two of the non-players talked about Friends who played games. These two non-players expressed frustration with the games; even though they did not understand the details of the games, both said they were "annoyed" by how their Friends were publishing game-related activity on their wall.

Only one participant said she played alone; all other players played with their Friends in cooperative or competitive ways. This was not surprising as most of the popular games on Facebook [3] are designed for social play.

Participants' Neighbors were extremely diverse, including close family members such as siblings and spouses; distant acquaintances such as far cousins and friends from high school; and complete strangers. The age range of game players that participants identified was wider than our participant pool: one participant talked about his grandmother, who he said used Facebook primarily to play games.

In some cases, participants became Friends with complete strangers through online forums or through mutual Friends. A couple of participants talked about actively meeting and seeking new people through online discussion boards. There were also participants who met future Friends while playing the game. For instance, certain games have chat functions that allow people to chat if they are playing at the same time. This does not mean that the two people are playing with each other, but that they happen to be logged in to the game server simultaneously. Players talked about meeting people through the chat function and later deciding to become Friends.

\subsection{Initial motivations}

Examining RQ1, participants described two different types of motivations for playing SNGs. Although there were a number of different initial motivations that participants identified, which included playing the games to pass time, this paper will direct attention to the social-focused initial motivations. Our research focuses on how SNSs can promote social interactions, and therefore we focus our attention on the motivations of our participants that address this.

First, some users began playing SNGs as a way to seek social interaction with existing members of their Friend network. For example, when asked what characteristics of SNGs he preferred over other types of games, one participant (P9) noted that he was an avid game player even before SNGs were available, but he played SNGs when he had the desire to interact with other people, saying, "There's a socializing aspect to it. I play my PlayStation when I want to be left alone."

Another participant talked about maintaining connections with distant friends: 
I have friends from other countries and it's nice to keep the connection going with them even if it's just playing Farmville. (P18)

The second social motivation for participants was to be considerate of Friends who were requesting them to join the game. A number of participants began playing games on Facebook after receiving these prompts, which often entailed the Friend needing more Neighbors in order to complete an ingame task. At times, participants noted that they gave in after repeated requests from a Friend just to get the requests to stop. One woman noted that her sister sent her requests for weeks before she joined:

I started getting into Mafia Wars probably two months ago, yeah. That was because of my sister. She kept sending me all this information and polluting my page [laughs]. I said "what are you doing?" [laughs] And I just kept ignoring her invites for like weeks... And then I just went on too because she needed more members so I just did it to shut her up... then I got hooked. (P3)

Facebook users also received game-related information, such as Friends' in-game achievements, through their News Feed:

When you're playing these games, it asks you if you want to post that you just made this accomplishment, or you just fertilized somebody's crops, you just did this, you just did that, and you can tell it yes, publish it on your wall or no. (P11)

However, for non game-players, these posts were particularly annoying:

Some of them just get really carried away with constant News Feed postings from those things. And to me, I don't need to see that. (P16)

I really hate all the applications. I've got a bunch of them hidden because I'm just tired of seeing them. (P2)

For others, however, the steady stream of status updates from their friends may have piqued their curiosity about the game, which may have made them more likely to join when asked by their Friends:

I saw constant updates about Farmville along with all the other million things that are out there like Café World and it seems like every day, there's a new one. And I ignored it at first... But [then] a friend did ask me, she said she needed one more neighbor... so I joined. (P8)

\subsection{Communication cues}

A SNS is a set of technological tools centered around the concept of a social network. These tools facilitate interaction by providing users with a number of ways through which to communicate both publicly (e.g., status updates, wall posts) and privately (e.g., inbox messages, chat). Participants talked about how they used multiple features of Facebook in addition to features of the SNGs in order to create, maintain, and enhance their social connections.

Our participants used multiple channels to communicate with Friends as part of their SNG play. The most direct type of communication occurred when two Friends were playing the game synchronously. Participants used instant messaging features, including both Facebook chat and in-game chat, and even used non-Facebook channels such as the telephone.

Although not synchronous, players were also communicating directly by commenting on each other's Walls. One participant, who said he met half of his Facebook Friends through Farmville, talked about how comments on his game status updates gave him social support:

When you're playing that game, if you hit like a bonus or if you go up a level or something, then somebody will comment on there, good job, or something like that, you know. (P1)

Not all social components of SNGs, however, were about direct interaction. Since the nature of the games allow asynchronous play, some participants passively obtained information about their Neighbors through the site-including high scores and in-game aesthetics, such as how they designed their farms and restaurants - and indirectly competed with their Neighbors by trying to get a higher score or design a better virtual space. Two participants commented on various aspects of friendly competition, which included comparing their virtual space decoration with others':

I look at people's levels, and then I try to beat theirs. So if I'm a 22, and I get on there and see my cousin's a 23, I'm gonna get that. I'll be there on all day 'cause I have to be 
higher than her. [With] some people...I look at their café and see how they decorated, and I try to re-decorate mine to make it look better. (P14)

I have one friend that has no animals or buildings or anything. He's just mass producing crops so he can just accelerate his level. He started after me and now he's like three times ahead of me... he's always teasing me that I look like I'm playing house with mine 'cause I've got the whole thing set up like a little farm, so there's some friendly competition on there, but I think with women it's just a way to play with a little dollhouse without somebody looking at you like you're crazy because you're a grown-up. (P9)

Even when SNGs did not allow for an in-game comparison of levels or aesthetics, the games still facilitated social interaction among some participants. For example, one participant noted that she and her husband, who was in the military, played games on Facebook and then compared scores by posting them to their walls. In this way, interaction between two Friends moved outside of the game and into the network more generally.

Participants also engaged in indirect communication by interpreting certain elements of game play as communicative cues. For instance, in Farm simulation games, some participants perceived in-game helping acts (e.g., fertilizing a Neighbor's crops) as a proxy for more general kindness. One participant explained that the grateful feeling was because the helping behavior was not required by the game, it carried more sentimental value:

You don't have to do it, so if you do, I think it's like a way saying you're interested in that person in a certain way. It's just like being kind, like holding a door open for somebody, you could just walk through and let it slam in their face or you could hold it open for them. So I think with the fertilizing [a Neighbor's crops], it's saying, "I care enough about your crops so that you can get some extra points and I can get some extra points." Yeah, another form of a friendly thing to do. (P8)

These helping behaviors - such as giving gifts or helping a Neighbor fulfill specific in-game taskscan also be reciprocated. One participant talked about reciprocity in the game Fish World:
You have Neighbors... and you can send them gifts for their aquarium and they can send gifts to your aquarium. They can steal your fish and you can steal their fish. You can work in their aquarium, they can work in yours. (P11)

Another participant talked about how reciprocity of the helping behaviors and virtual item exchange gave him a sense of belonging to a larger group:

You have this kind of backdrop of, "Here's all the other people who play this too," and you have this kind of community of people that know each other that are collaborating... With these games, you can send gifts to one another and help each other out. (P7)

\section{Relationships}

The social elements fostered in SNG play naturally created different relationship dynamics among game players. Although the activities participants engaged in were similar, the game play affected their relationships differently depending on with whom they were playing.

\subsection{Initiating}

Many participants talked about initiating new relationships with people they did not previously know. SNG players who initiated new relationships for game-relate purposes were typically interested in the game play as much as (or even more than) the social components involved in playing, as indicated by several participants who emphasized that they tried to increase the size of their game network for the sake of advancing their game level. This motivation is related to the mechanics of the game, which provides advantages to players that have more game Neighbors. One participant explains:

You can get farther in the game if you have Neighbors, and Neighbors have to already be your friend on Facebook. You can't just ask somebody that plays the game that you don't know, or that's not your friend on Facebook to be your Neighbor. You have to ask one of your friends to be your Neighbor. (P11)

Although many people were just playing with close friends and family members, some SNG 
players went out of their way to find people from outside of their Friend network. One participant, who became Friends with people around the world, talks about the process in which she found a new Friend and game Neighbor through a blog:

Mafia Wars just started a blog and so you can go and click on there and see who they are on Facebook and send 'em like a message. So there's this guy, he was saying something, and I just sent him a little comment about what he was saying, that kind of stuff. (P4)

Participants also talked about how they obtained Neighbors through "Friends of Friends." In many cases, a mutual Friend would suggest that the two people become Friends so they could both benefit from having more Neighbors in the game:

I just recently had a Neighbor on there contact me to become friends with her friends so that we could all become Neighbors on Farmville so that we could get more ribbons. So I just got 4 new Neighbors and friends at the same time. (P8)

A couple days ago, I was working on this guy's farm and he posted he needed Neighbors and was anybody interested in getting more Neighbors. And I said that I needed some Neighbors, cause I need ten Neighbors to make my farm bigger, and I only had eight. [laughs] So I was like, "yeah, sure, I'll be your Neighbor!" So that's how I gained a friend and a Neighbor at the same time, isn't that just wonderful? (P11)

While Friending for gaming purposes appeared to be a primary motivation for several participants, participation in the game also facilitated relationship development by revealing common interests unrelated to the game. For example, one participant (P9) talked about how he gained a new Neighbor by discussing his interests with other players while playing the game Camelot. He was talking to his Friend about artwork in general and how he liked scenic paintings, so the Friend introduced him to another Camelot player who was an artist of scenic paintings.

In yet another example, the man discusses how he met someone new through a discussion based on comments posted on a Friend's photo:

P: A friend of mine at work, her husband caught a huge fish. She posted a picture of it and said, "Look what my husband did." I posted a comment asking what kind of fish it was, 'cause I didn't know. He started answering me and then we started talking about that and even other things.

I: And did you end up Friending him or not? $P$ : Actually, he's one of the people I play the game with. (P9)

SNGs also served as a reason for distant relatives to connect with each other. One man talked about how he used Farmville as a method to become Neighbors with a relative he had never met in person.

She is the wife of my first cousin once removed. [laughs] So I've never met her and I probably never will. We've chatted a tiny bit just to sort of say " $h i$ " and "life is good" sorta things, but she's a Friend on Farmville. (P18)

\subsection{Maintaining}

For people who were playing with Friends they knew before joining the game, one of the reasons for being Neighbors was to maintain these existing relationships. Eight participants talked about how SNGs were one of multiple methods through which they maintained existing relationships through Facebook. Participants who used games as a relationship maintenance tool talked about how the games were a method of connecting with friends and family members who were geographically distant. One woman (P12) talked about using the gifting feature in Facebook games as a means of keeping in touch with her sisters, who currently live in France.

Another woman talked about using games as to stay more connected with her family, who she didn't get to see in person very often because they lived in a different state:

I live in Virginia, and all my family lives in Maryland. So that's really the only time I get to talk to them, to play games with them, 'cause we live so far away. (P14)

Playing games together may also help establish common ground between individuals. One man noted that playing games with his Friends benefited their relationships by giving them something small that they could share with each other on a regular basis: 
Well, a lot of my friends live out of state, so I feel that if we work toward a common goal like killing the monster or whatever, it gives us something that we still have in common, even though I don't see them every day. I know that probably sounds silly, 'cause it's only a game, but I don't know, through a superficial long distance way of communicating, it still gives us the opportunity to stay connected somehow and that's important. (P10)

In addition to situations when individuals used the game to span geographical distances, two participants specifically noted that they played SNGs with their significant other. One man (P18) said he only played for the sake of his partner, and that he sends in-game gifts every now and then just for the sake of their offline relationship, while a female participant (P14) talked about friendly competition with her husband.

One of the reasons participants used these games as a means of communication instead of other communication channels (e.g., phone calls, emails) appears to be related to the low communication costs associated with in-game exchanges. For example, fertilizing a Neighbor's crops or visiting their café takes only a few seconds and can be completed as part of a regular visit to the game. A few participants expressed the game-play as being "free" and something that is "easy" to do. One man associated gift exchanges in SNGs as being low in costs:

[In] some of the other little goofy games like Farmville, Farm Town, I'll send invites or send people little gifts just to say "Hey, thinking about you." It doesn't cost anything. [laughs] I'm cheap. (P10)

\subsection{Enhancing}

Finally, some players used SNGs as a springboard for enhancing their relationships with Neighbors. This is most likely because SNGs provide an environment that facilitates conversation. Many participants commented on how playing games increased the depth of a relationship that was otherwise superficial. This mostly occurred among people who were Friends, but not very close:

I've definitely made friendships with some people that were just acquaintances; now we talk a lot more. We were already friends, but we just hooked up because we went to school together and then never really communicated.
Then we both ended up on Farmville and now we're talking back and forth. (P6)

Our participants also described the ways in which becoming a Neighbor with someone they didn't know sometimes led to actual online friendship. Much of this was driven by the fact that Neighbors had to first become Friends on Facebook, which subsequently gave them access to each other's profile on the site and added their posts to the News Feed. One participant talked about how his relationships with Neighbors he initially Friended for game-related purposes evolved into something more:

You can send messages. You can discuss things with them. It's a very interactive type of a game. You see everything that's going on, you know, just like your normal friend on Facebook. So we comment on all kinds of things in people's life, not just Mafia Wars. Not anymore. (P4)

\section{Discussion}

As our findings suggest, participants' motivation of the initial Friending was mainly driven by the intent of increasing their game level. However, regardless of why they started playing the game, participants talked about how the game play affected their relationships. For example, although some participants added Friends as Neighbors because of the benefits that they would accrue through the game, they also experienced stronger relationships with their Neighbors as a result of playing the game. Although we cannot generalize that playing SNGs always creates positive outcomes, we can still find evidence that in certain circumstances, even "selfish" intentions can still lead to some kind of unintended positive social outcome.

Participants gave strong examples of building both bridging and bonding social capital through the games. Supporting previous research that suggests that SNSs have unique affordances due to the low transactions costs associated with maintaining a large network [10], participants were using their existing ties to expand their network to include more game players through mutual connections. Forging friendships through mutual connections may also be an example of what Haythornthwaite [15] describes as "latent ties." Latent ties are potential connections that have not been socially activated yet. Ellison et al. [12] suggest that Facebook's inclusion of a wide range 
of identifying information such as mutual friends and share interests may encourage users to activate latent ties. Our data suggest that this was taking place through games on the site.

Participants talked about how cooperative game play had a positive effect on their relationships. Reciprocity, fostered through the exchange of ingame gifts and helping behavior, could be a key mechanism of SNGs that supports relationship maintenance. We found that some participants were committed to helping others in the games. Although they engaged in reciprocity because it was relatively easy act that would help them increase their game level, they were appreciative when others were doing it for them. Future research should see if certain types of reciprocity have different effects on relationships and the differences between quantity and quality.

Previous game studies have mostly focused on negative effects such as aggression [1,19] and the harmful effects that playing games may have on social development and self-esteem [2]. However, the relationship maintenance behavior that SNGs facilitate suggests that players are getting positive outcomes from playing the game. Although we are not implying that playing SNGs will always lead to prosocial results, there are indicators that certain social interactions have a positive effect on relationships outside of the game.

Previous research on MMOs has shown that design elements of the game sometimes strip away reason to converse with other players [11]. Future research should try to identify which interactions actually contribute to the prosocial effects and how the design of the medium plays a role in facilitating those interactions.

Our study suggests that social games can make it easier to enhance relationships by giving people a common interest to discuss, an easy-to-use place to discuss this and other topics, and incidental exposure via the news feed to other aspects of the lives of people they may not normally interact with much. Because many SNGs require interaction between players, it could be that the games act as a context for communication outside of the game, which deepens the connections between the players. Future research can look at SNSs more generally to determine how prevalent this activity is, and how strong an effect there is on the relationships between players.

\section{Conclusion}

In this study, we examine the social interactions that occur through social network games (SNGs)games that are embedded within social network sites such as Facebook-through interviews with
Facebook users who were SNG players or who had Friends who were SNG players.

Social factors are extremely important in getting people to start games in the first place. Even if game play doesn't facilitate direct social interaction, indirect interaction and using the game as a topic of common interest can be useful in maintaining and even enhancing relationships.

\section{Acknowledgments}

This work was supported in part by the National Science Foundation (HCC 0916019).

\section{References}

[1] Anderson, C. et al., "Longitudinal effects of violent video games on aggression in Japan and the United States", Pediatrics, 122(5). 2008. e1067

[2] Anderson, C. and B. Bushman, "Effects of violent video games on aggressive behavior, aggressive cognition, aggressive affect, physiological arousal, and prosocial behavior: A meta-analytic review of the scientific literature", Psychological Science, 12(5). 2001.

[3] AppData, "Independent, Accurate Facebook Application Metrics and Trends from Inside Facebook", Available from: http://www.appdata.com/leaderboard/

[4] Bartle, R., "Players who suit MUDs", Journal of MUD Research, 1(1), 1996.

[5] Burke, M., C. Marlow, and T. Lento. "Social network activity and social well-being". In Proceedings of the ACM Conference on Human Factors in Computing Systems, 2010. Atlanta, GA.

[6] Chen, J. et al., "Make new friends, but keep the old: recommending people on social networking sites", in Proceedings of the ACM Conference on Human Factors in Computing Systems, 2009. Boston, MA

[7] Chen, M., "Communication, coordination, and camaraderie in World of Warcraft", Games and Culture, 4(1), 2009.

[8] Cole, H. and M. Griffiths, "Social interactions in massively multiplayer online role-playing gamers". CyberPsychology \& Behavior, 10(4). 2007. 575-83

[9] Corbin, J. and A. Trauss, "Basics of qualitative research: Techniques and procedures for developing grounded theory", Sage Publications, 3rd Edition, 2007.

[10] Donath, J. S. and d. boyd, "Public displays of connection", BT Technology Journal, 22(4), 2004. 71

[11] Ducheneaut, N. and R. Moore, "The social side of gaming: a study of interaction patterns in a massively multiplayer online game", in Proceedings of the ACM 
conference on computer supported cooperative work (CSCW), Chicago, IL, 2004.

[12] Ellison, N. B., C. Steinfield, and C. Lampe, "The benefits of Facebook "friends: "Social capital and college students' use of online social network sites". Journal of Computer-Mediated Communication, 12(4), 2007.

[13] Gross, D. "The Facebook games that millions love (and hate)", [cited 2010 Feb. 8]; Available from: http://articles.cnn.com/2010-02-

23/tech/facebook.games_1_mark-pincus-video-gamesfacebook?_s=PM:TECH

[14] Hargittai, E. "Whose Space? Differences Among Users and Non-Users of Social Network Sites", Journal of Computer Mediated Communication, 13(1), 2007.

[15] Haythornthwaite, C. "Social Networks and Internet Connectivity Effects". Information, Communication \& Society, 8(2), 2005. 125-47

[16] Joinson, A. N. "Looking at, looking up or keeping up with people?: Motives and use of Facebook", in Proceedings of the ACM Conference on Human Factors in Computing Systems, 2008. Florence, Italy.

[17] Kirman, B. et al. "Improving social game engagement on Facebook through enhanced socio-contextual information", in Proceedings of the ACM Conference on Human Factors in Computing Systems, 2010. Atlanta, GA.

[18] Lampe, C., N. Ellison, and C. Steinfield. "A Face(book) in the crowd: Social searching vs. social browsing", in ACM Conference on Human Factors in Computing Systems, 2006. New York: ACM.

[19] Lee, K. M. and W. Peng, eds. "What do we know about social and psychological effects of computer games? A comprehensive review of the current literature", in Playing video games: motives, responses, and consequences, 2006. Lawrence Erlbaum Associates.

[20] Lenhart, A., K. Purcell, A. Smith, and K. Zickhur, "Social media \& mobile internet use among teens and young adults", 2010. Pew Internet and American Life Project.

[21] Lewis, J. and A. West, “'Friending': London-based undergraduates' experience of Facebook", New Media \& Society, 11(7). 2009. 1-21

[22] McCarthy, C., "'Scrabulous' debate may rewrite the rules of the game", Cnet, January 16, 2008, Retrieved from http://news.cnet.com/8301-13577_3-9852054-36.html

[23] Miles, M. B. and M. Huberman, "Qualitative data analysis: An expanded sourcebook", Sage Publications, 2nd edition, Thousand Oaks, CA, 1994
[24] Onwuegbuzie, A. J. and N. L. Leech, "Validity and qualitative research: An oxymoron?" Quality and Quantity, 41, 2007.

[25] Papacharissi, Z. and A. Mendelson, "Friends, networks and zombies: The social utility of Facebook", in Annual Meeting of the Association of Internet Researchers, 2008. Copenhagen, Denmark.

[26] Raacke, J. and J. Bonds-Raacke, "MySpace and Facebook: Applying the uses and gratifications theory to exploring friend-networking sites", CyberPsychology \& Behavior, 11(2), 2008, pp. 169-74.

[27] Resnick, P., "Beyond bowling together: SocioTechnical capital", in Human-computer interaction in the new millennium, ed. J.M. Carroll, Addison Wesley, Reading, MA, 2001.

[28] Selwyn, N. "Faceworking: exploring students' education-related use of Facebook", Learning, Media and Technology, 34, 2009. 157-74

[29] Sherry, J. L. and K. Lucas, "Video game uses and gratifications as predictors of use and game preference" in Playing video games: motives, responses, and consequences, ed. P. Vorderer and J. Bryant, Routledge, New York, 2006.

[30] Skeels, M. and J. Grudin. "When social networks cross boundaries: a case study of workplace use of facebook and linkedin." In Proceedings of the ACM International Conference on Supporting Group Work, Sanibel Island, FL, 2009, pp. 95-104.

[31] Steinfield, C., N. B. Ellison, and C. Lampe, "Social capital, self-esteem, and use of online social network sites: A longitudinal analysis", Journal of Applied Developmental Psychology, 29, 2008, pp. 434-45.

[32] Steinfield, C., J. M. DiMicco, N. B. Ellison, and C. Lampe, "Bowling online: Social networking and social capital within the organization", in Fourth International Conference on Communities and Technologies, 2009: New York: ACM.

[33] Williams, D., "Why game studies now? Gamers don't bowl alone", Games and Culture, 1(1), 2006.

[34] Williams, D., N. Ducheneaut, L. Xiong, Y. Zhang, N. Yee and E. Nickell, "From tree house to barracks: The social life of guilds in World of Warcraft", Games \& Culture, 1(4), 2006, pp. 338-361.

[35] Wohn, D., Y. Lee, J. Sung, and T. Bjornrud, "Building common ground and reciprocity through social network games", in Proceedings of ACM Conference on Human Factors in Computing Systems,2010. Atlanta, GA.

[36] Yee, N., "Motivations for play in online games", CyberPsychology \& Behavior, 9(6), pp. 772-75, 2006. 